\title{
Vimentin regulation of autophagy activation in lung fibroblasts in response to lipopolysaccharide exposure in vitro
}

\author{
Pan Pan ${ }^{1}$, Longxiang Su ${ }^{2}$, Xiaoting Wang ${ }^{2}$, Wenzhao Chai ${ }^{2}$, Dawei Liu ${ }^{2}$, Licheng Song ${ }^{1}$, Lixin Xie ${ }^{1}$ \\ ${ }^{1}$ College of Pulmonary and Critical Care Medicine, Chinese PLA General Hospital, Beijing, China; ${ }^{2}$ Department of Critical Care Medicine, State \\ Key Laboratory of Complex Severe and Rare Disease, Peking Union Medical College Hospital, Peking Union Medical College, Chinese Academy of \\ Medical Sciences, Beijing, China \\ Contributions: (I) Conception and design: P Pan, L Su; (II) Administrative support: L Su, P Pan; (III) Provision of study materials or patients: L Xie, \\ D Liu; (IV) Collection and assembly of data: L Song; (V) Data analysis and interpretation: X Wang, W Chai; (VI) Manuscript writing: All authors; (VII) \\ Final approval of manuscript: All authors. \\ Correspondence to: Longxiang Su. Department of Critical Care Medicine, Peking Union Medical College Hospital, Peking Union Medical College, \\ Chinese Academy of Medical Sciences, 1 Shuaifuyuan, Dongcheng District, Beijing 100730, China. Email: sulongxiang@vip.163.com; Lixin Xie. \\ College of Pulmonary and Critical Care Medicine, Eighth Medical Center of Chinese PLA General Hospital, 17th Heishanhujia, Haidian District, \\ Beijing 100091, China. Email: xielx301@126.com.
}

\begin{abstract}
Background: The activation and assembly of the NLRP3 inflammasome is dependent on the interaction between NLRP3 and the intermediate filament protein vimentin in an acute respiratory distress syndrome (ARDS) model. We investigated the role of vimentin in this process using human fetal lung (HFL-1) fibroblasts with vimentin transfer genes or gene knockdown and lipopolysaccharide (LPS) intervention.

Methods: HFL-1 cells [con-vector + LPS, vimentin-pCMV3 (VIM-pCMV3), con-siRNA, and vimentin siRNA (VIM-siRNA)] were treated with LPS. An oxidative stress damage assessment, apoptosis analysis, and quantification of tumor necrosis factor- $\alpha$ (TNF- $\alpha$ ), interleukin (IL)-1 $\beta$, IL-6, and IL-10 by enzyme linked immunosorbent assay (ELISA) were performed. Immunoblotting was used to reveal the autophagy pathway.

Results: We demonstrated that in response to LPS vimentin expression was lower in the HFL-1 cells with the vimentin gene knocked down. Specifically, an increase in oxidative stress, a decrease in mitochondrial membrane potential, or an increase in calcium ion permeability resulted in an increase in the fibroblast apoptosis rate. In addition, the inflammatory response after vimentin gene knockout was upregulated, as indicated by higher levels of TNF-a, IL-1 $\beta$, IL-6, and IL-10. Importantly, the mechanism of suppression of vimentin in the lung fibroblasts was caused by a decrease in autophagy, an increase in mitochondrial membrane protein, and a decrease in mitochondrial function, which may contribute to the augmented cellular injury generated during the response to LPS.

Conclusions: This study provides insights into whether vimentin may interfere with the inflammatory cascade by activating the autophagy pathway of mitochondrial lung fibroblasts in the early stage of acute lung injury (ALI).
\end{abstract}

Keywords: Vimentin; human fetal lung-1 cells (HFL-1 cells); acute respiratory distress syndrome (ARDS); inflammation; mitochondrial autophagy

Submitted Jul 03, 2020. Accepted for publication Nov 20, 2020.

doi: 10.21037/atm-20-5129

View this article at: http://dx.doi.org/10.21037/atm-20-5129

\section{Introduction}

Sepsis is a serious systemic inflammatory state that is associated with the presence of a known or suspected infection, often with rapid onset. Patients with sepsis are usually treated in the intensive care unit, administered intravenous fluids and antibiotics, and possibly mechanical ventilation to support lung function. Acute lung injury (ALI) and acute respiratory distress syndrome (ARDS) often occur in sepsis. The annual incidence 
of ALI/ARDS in the United States is approximately 200,000 cases, with estimated mortality rates ranging from $25 \%$ to $60 \%$ (1). Progressive ALI occurs in one-third of patients with sepsis. ALI/ARDS is an extraordinarily complex lung disorder characterized by a neutrophilic inflammatory response and is associated with increased pulmonary vascular permeability. This destruction leads to pulmonary edema, intrapulmonary hemorrhage, and severely impaired gas exchange. The pathophysiology of ALI/ARDS in sepsis is complex and multifactorial and includes proinflammatory cytokines, chemokines, and reactive oxygen species (ROS) $(2,3)$. Despite extensive research and progress in several fields of ALI/ARDS, the appropriate therapeutic interventions are still limited, and the mortality of patients who develop septic ALI/ARDS remains unacceptably high. Although the precise mechanisms underlying the development of ALI/ARDS following sepsis remain unclear, the inflammatory response is considered a major contributor $(4,5)$.

Vimentin, a type III intermediate filament involved in the motility and maintenance of cell shape, has also been implicated in inflammatory responses. Apart from being a cytoskeletal protein, its alternative roles in cell biology are gaining interest. Vimentin is associated with macrophage differentiation, phagocytosis and ROS production. Vimentin is expressed on activated macrophages and is secreted in response to proinflammatory stimuli $(6,7)$. A recent study demonstrated that the activation and assembly of the NLRP3 inflammasome is dependent on the interaction between NLRP3 and the intermediate filament protein vimentin (8). In line with our search for biomarkers useful for sepsis diagnosis and prognosis, we explored protein components by proteomics in blood samples collected from patients with sepsis or septic shock and found that serum vimentin was significantly increased in these patients (9). Furthermore, we experimentally verified that vimentin can regulate the expression of apoptosis and cytokines in lymphocytes and monocytes $(9,10)$. Therefore, we aimed to determine whether vimentin is required for inflammasome activation and lung function in an HFL-1 cell model of LPS-induced lung injury. We present the following article in accordance with the MDAR reporting checklist (available at http://dx.doi.org/10.21037/atm-20-5129).

\section{Methods}

\section{Cell culture and treatment}

Human fetal lung (HFL-1) fibroblasts were purchased from American Type Collection of Culture (Manassas, VA, USA) and cultured in DMEM supplemented with $10 \%$ fetal calf serum (FCS), $50 \mathrm{U} / \mathrm{mL}$ penicillin/streptomycin and $50 \mathrm{mg} / \mathrm{mL}$ fungizone. The cells were divided every $3-5$ days. The cells were treated under the following eight conditions: cells transfected with control vector (con-vector), vimentin-expressing vimentinpCMV3 vector (VIM-pCMV3), control siRNA (con-siRNA), or vimentin-specific siRNA (VIM-siRNA), and the cells of these four conditions plus lipopolysaccharide (LPS) exposure (con-vector + LPS, VIM-pCMV3 + LPS, con-siRNA + LPS, and VIM-siRNA + LPS). For LPS intervention, the cells were treated with $1 \mu \mathrm{g} / \mathrm{mL}$ LPS [derived from O55:B5 Escherichia coli (E. coli); Sigma, USA] and incubated for 48 hours as described in a previous publication $(11,12)$.

\section{Transfection of cells with siRNA and plasmids}

For siRNA transfection, HFL-1 cells were plated into $60-\mathrm{mm}$ tissue culture dishes $\left(3 \times 10^{6} \mathrm{cells} / \mathrm{dish}\right)$ and cultured overnight. The next day, the cells were washed once with SF-DMEM without antibiotics and amphotericin B followed by treatment with $1 \mathrm{~mL} /$ dish Opti-MEM. Negative control siRNA (con-siRNA, Santa Cruz Biotechnology, Cat\#: sc-108060) or vimentin-specific siRNA (VIM-siRNA, Santa Cruz Biotechnology, Cat\#: sc-29522) were mixed with Lipofectamine 2000 (Santa Cruz Biotechnology, Cat\#: sc-29528) in Opti-MEM following the manufacturer's instructions. The mixture was then added to the $60-\mathrm{mm}$ dish containing cells $(500 \mu \mathrm{L} / \mathrm{dish}$, final concentration of siRNA was $200 \mathrm{nM}$ in a $1.5 \mathrm{~mL} /$ dish). After $6 \mathrm{~h}$ of transfection, the cells were further cultured for $24 \mathrm{~h}$ with $10 \%$ FCS-DMEM supplemented with antibiotics and amphotericin $\mathrm{B}$ overnight. Cells were then used for experiments as designed.

Plated HFL-1 cells that transfected with vimentinoverexpressing plasmid into $60 \mathrm{~mm}$ tissue cultures $\left(3 \times 10^{6}\right.$ cells/dish $)$ and cultured overnight. The next day, the cells were washed once with SF-DMEM without antibiotics and amphotericin B followed by the addition of $1 \mathrm{~mL}$ Opti-MEM. The vimentin-expressing plasmid (pCMV3VIM, SinoBiological Inc., Beijing, China) or an untagged negative control vector (pCMV3-untagged negative control vector) was mixed with the transfection reagent (Sinofection-293, Sino Biological Inc., Beijing, China) in Opti-MEM following the manufacturer's instructions. The mixture was then added to the $60 \mathrm{~mm}$ dish containing the cells $(500 \mu \mathrm{L} /$ dish, with a final concentration of siRNA of $200 \mathrm{nM}$ in $1.5 \mathrm{~mL} /$ dish). After $6 \mathrm{~h}$ of transfection, the cells were further cultured for $24 \mathrm{~h}$ with $10 \%$ FCS-DMEM 
supplemented with antibiotics and amphotericin $\mathrm{B}$ overnight. Cells were then used for experiments as designed.

\section{Immunofluorescence staining}

The HFL-1 cells were fixed with 4\% paraformaldehyde, rinsed three times in phosphate buffer saline (PBS) for $5 \mathrm{~min}$ each time, and then incubated with $0.1 \%$ Triton $\mathrm{X}-100$ for $10 \mathrm{~min}$ at room temperature. After washing three times for $5 \mathrm{~min}$ each, the cells were blocked with $10 \%$ bovine serum for $30 \mathrm{~min}$ and then incubated overnight with FITC-conjugated mouse anti-human vimentin (ab128507, Abcam, Cambridge, Massachusetts) at a 1:200 dilution at $4{ }^{\circ} \mathrm{C}$. After three rinses in PBS, the cells were incubated with 594-conjugated Affinipure donkey anti-rabbit IgG (H + L) (Jackson Immuno Research Inc., PA, USA) at a 1:400 dilution in bovine serum albumin (BSA)-phosphate buffered saline (PBS) for $30 \mathrm{~min}$ at $37^{\circ} \mathrm{C}$. After washing, the labeled samples were examined using a confocal microscope (Leica Microsystems GmbH, Germany).

\section{Reverse transcription-polymerase chain reaction (RT-PCR)}

Total RNA was extracted from the samples using TRIzol reagent (Invitrogen, Carlsbad, CA, USA). Reverse transcription was performed using SuperScript III (Invitrogen). Real-time PCR was performed using sequence-specific primers: vimentin-F 5'-GTCTCTGGCACGTCTTGAC-3', vimentin-R: 5'-TGCTGTTCCTGAATCTGAGC-3'; GAPDH-F: 5'-GCCTTCCGTGTCCCCACTGC-3', GAPDH-R: 5'-GGCTGGTGGTCCAGGGGTCT-3'. PCR was performed using an Eppendorf 5333 Master Cycler thermocycler (Eppendorf, lot: 5333 53658) and Eppendorf Master cycle reprealplex (Eppendorf, lot no. X226488N) with an initial denaturing step at $95{ }^{\circ} \mathrm{C}$ for $15 \mathrm{sec}, 40$ cycles of denaturing at $95{ }^{\circ} \mathrm{C}$ for $30 \mathrm{sec}$ and annealing at $55^{\circ} \mathrm{C}$ for $20 \mathrm{sec}$. Vimentin gene expression, which was normalized to GAPDH expression, was analyzed by the $\Delta \Delta \mathrm{Ct}$ method (13).

\section{Detection of oxidative stress damage}

Detection of oxidative stress damage included measurements of ROS, changes in mitochondrial membrane potential, and calcium accumulation. ROS were measured with a dihydroethidium fluorescence probe (Catalog \#KGAF019, KeyGen Biotech, Nanjing, China). Mitochondrial membrane potential and calcium accumulation were detected using a JC-1 mitochondrial membrane potential assay kit (Catlog\#10009172, Cayman, Ann Arbor, MI, USA) and Fluo-4 AM assay kit (Catalog \# S1060, Beyotime Biotechnology, Shanghai, China). All these detection experiments were conducted following the manufacturers' instructions. Fluorescence changes in the HFL-1 cells were detected, photographed, and counted in at least 5 random fields of each slide with a fluorescence microscope at $200 \times$ magnification (Leica, Germany).

\section{Apoptosis and FACS analysis}

Cells $\left(2 \times 10^{5}\right.$ cells $/ \mathrm{ml} /$ well, $\left.\mathrm{n}=3\right)$ were seeded in 24 -well plates and stimulated with LPS for 6,12 or $24 \mathrm{~h}$. The cells were harvested using $0.25 \%$ trypsin-EDTA at $37{ }^{\circ} \mathrm{C}$. The cells were stained with an Annexin V-EGFP apoptosis detection kit (KeyGen Biotech Co., Ltd., China) following the manufacturer's instructions and analyzed with a flow cytometer (BD Accuri ${ }^{\mathrm{TM}}$ C6 Plus flow cytometer, Accuri Cytometers Inc., Cambridge, UK). The apoptosis rate for each condition was measured by flow cytometry at least 3 times.

\section{Quantification of tumor necrosis factor- $\alpha(T N F-\alpha), I L-1 \beta$, IL-6, and IL-10 by ELISAs}

To assess the effect of vimentin on fibroblast release of TNF- $\alpha, \mathrm{IL}-1 \beta$, IL- 6 , and IL-10, HFL- 1 cells were plated in monolayers $\left(2 \times 10^{5}\right.$ cells $/ \mathrm{mL}, 1 \mathrm{~mL} /$ well of a 12 -well plate $)$ in $10 \%$ FCS-DMEM and cultured for 2 days. The different groups of cells were then treated with $1 \mathrm{ml} /$ well serum-free DMEM (SF-DMEM). After $24 \mathrm{~h}$ of treatment, the medium was harvested for the quantification of cytokines by enzyme linked immunosorbent assays (ELISAs) (SEA133Hu; SEA563Hu SEA079Hu; SEA056Hu, Wuhan USCN Business Co., Ltd., China) following the manufacturer's instructions.

\section{Immunoblotting}

The cells were lysed with ice-cold cell lysis buffer. The protein concentration was then determined using a butyleyano acrylate (BCA) protein assay kit (Applygen Gene Technology Corp, Beijing). Protein samples (100 $\mu \mathrm{g} / \mathrm{lane})$ were separated by $10 \%$ SDS-PAGE and then transferred onto nitrocellulose membranes. After blocking with 5\% skimmed milk powder in TBST (T: Tris; B: Buffer; S: Solution; T: Tween), the membranes were incubated with the following primary antibodies: anti-vimentin (Abcam cat. ab128507, 1:500 dilution), anti-COXII (CST cat.4842, 1:500 dilution), 


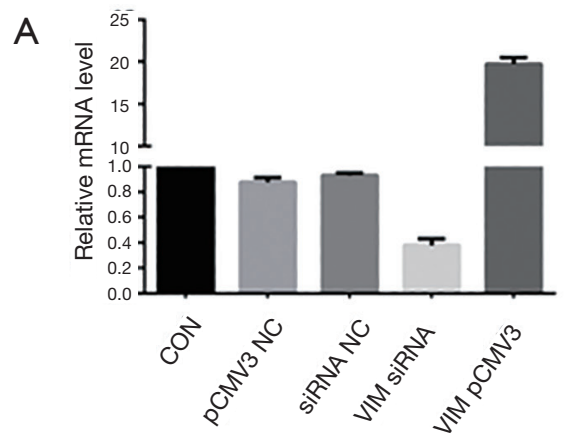

B

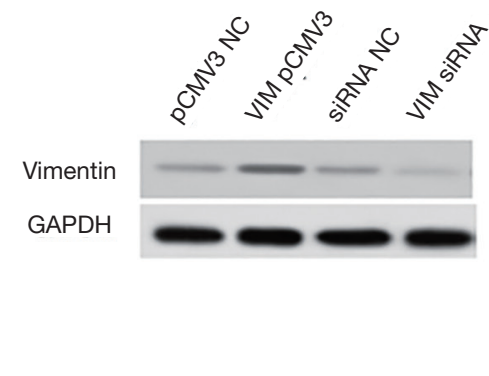

Figure 1 Vimentin expression in HFL-1 cells. HFL-1 cells were transfected with an untagged negative control vector (pCMV3-NC), a plasmid expressing vimentin (VIM-pCMV3), control siRNA (siRNA NC), and vimentin siRNA (VIM-siRNA) as described in the methods section. Representative RT-PCR (A) and immunoblotting (B) demonstrated either the overexpression or suppression of vimentin by VIM-pCMV3 or VIM-siRNA, respectively.

anti-TOM20 (BD cat.612278, 1:1,000 dilution), anti-TIM23 (BD cat.611223, 1:500 dilution), anti-Bcl-2 (CST cat.3498, 1:500 dilution), anti-caspase-3 (CST cat.9962, 1:500 dilution), and anti-P62 (MBL cat.PM045, 1:1,000 dilution), and anti-LCII (MBLcat.PM036, 1:1,000 dilution) and incubated overnight at $4{ }^{\circ} \mathrm{C}$. After washing, peroxidase-conjugated secondary antibodies were used. The protein bands were visualized by the electrochemiluminescence (ECL) kit. The intensities of the protein bands were analyzed by Gel-Pro 3.2 software. GAPDH (CST cat.5174, 1:1,000 dilution) was used as the internal control.

\section{Statistical analysis}

All data are expressed as the means \pm SEM. Statistical comparisons of multigroup data were analyzed by ANOVA followed by Bonferroni's (two-way) or Tukey's (one-way) posttest correction using PRISM4 software.

\section{Results}

\section{Vimentin expression in response to LPS}

PCR and western blotting were performed to demonstrate vimentin gene and protein expression in HFL-1 cells after transfection with the pCMV3 vector and siRNA (Figure 1). Both the relative mRNA (relative mRNA level) and protein level (protein ratio vs. control) assays revealed that vimentin was significantly increased in the cells transfected with the VIM-pCMV3 vector but was decreased in the cells transfected with VIM-siRNA (con vs. VIM-pCMV3 vector vs. VIM-siRNA, $\mathrm{P}<0.05)$. These results demonstrated that successful overexpression or suppression of vimentin in the HFL-1 cells was achieved.

As shown in Figure 2, LPS stimulated vimentin expression in the HFL-1 cells, as evidenced by a more intense immunofluorescence than that of the control group (con vs. LPS group, $\mathrm{P}<0.05$ ). Vimentin expression was further augmented by LPS in the cells transfected with the VIM-pCMV3 vector (LPS group vs. LPS + VIMpCMV3 vector group, $\mathrm{P}<0.001)$, while it was attenuated in the cells transfected with VIM-siRNA even in the presence of LPS (LPS group vs. LPS + VIM-siRNA group, $\mathrm{P}<0.001)$. However, there were no differences between the control group, VIM-pCMV3 vector control group, and VIM-siRNA control group $(\mathrm{P}>0.05)$.

\section{Oxidative stress and its damage}

Measures of ROS, mitochondrial membrane potential, calcium accumulation, and apoptosis were used here to evaluate the oxidative stress damage in the HFL-1 cells following LPS exposure. As shown in Figure 3A, ROS was significantly increased in response to LPS (con vs. LPS, $\mathrm{P}<0.05$, Figure $3 A, 1$ and 2), which was further increased in the cells transfected with VIM-siRNA (LPS alone vs. LPS + VIM-siRNA, $\mathrm{P}<0.05$, Figure $3 A, 1$ and 2) but significantly reduced in the cells transfected VIM-pCMV3 (LPS alone vs. LPS + VIM-pCMV3, $\mathrm{P}<0.05$, Figure $3 A, 1$ and 2). The mitochondrial membrane potential was significantly decreased in response to LPS (con vs. LPS, $\mathrm{P}<0.05$, Figure $3 B, 1$ and 2), which was further decreased in the cells transfected with VIM-siRNA (LPS alone $v s$. LPS + VIM- 

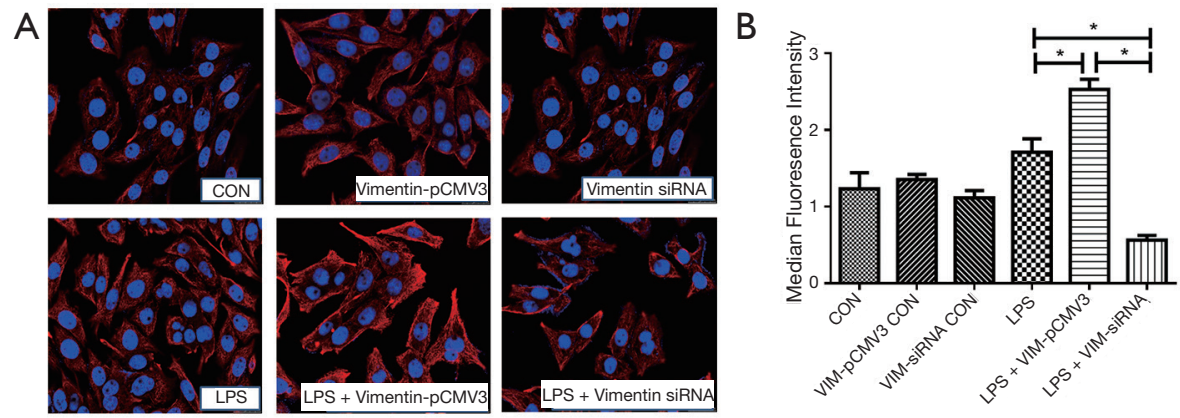

Figure 2 Vimentin expression (A) and fluorescence intensity (B) in the HFL-1 cells as visualized by immunoconfocal microscopy under a water immersion lens (400x). Red staining by the vimentin antibody reveals sites where vimentin is located. Blue spots show DAPI-stained cell nuclei. ${ }^{*} \mathrm{P}<0.05$.

siRNA, $\mathrm{P}<0.05$, Figure 3B, 1 and 2) but significantly blocked in the cells transfected with VIM-pCMV3 (LPS alone vs. LPS + VIM-pCMV3, $\mathrm{P}<0.05$, Figure 3B, 1 and 2). Similarly, the intracellular calcium concentration and apoptosis rate were significantly increased for the cells treated with LPS (calcium concentration: con vs. LPS, $\mathrm{P}<0.05$, Figure $3 C, 1$ and 2; apoptosis: con vs. LPS, $\mathrm{P}<0.05$, Figure $3 D, 1$ and 2), which were further increased in the cells transfected with VIM-siRNA (calcium concentration: LPS vs. LPS + VIM-siRNA, $\mathrm{P}<0.05$, Figure 3C, 1 and 2; apoptosis: LPS vs. LPS + VIM-siRNA, $\mathrm{P}<0.05$, Figure 3D, 1 and 2) but significantly decreased in the cells transfected with VIM-pCMV3 (calcium concentration: LPS vs. LPS + VIM-pCMV3, $\mathrm{P}<0.05$, Figure $3 C, 1$ and 2; apoptosis: LPS vs. LPS + VIM-pCMV3, $\mathrm{P}<0.05$, Figure 3D, 1 and 2). There were no significant differences between the three groups in terms of ROS, JC-1, or calcium concentration (all $\mathrm{P}>0.05$ ). However, there were significant differences in the calcium concentration and apoptosis rate for the control and VIM-pCMV3/VIM-siRNA groups (all $\mathrm{P}<0.05$ ).

\section{Release of inflammatory cytokines}

TNF- $\alpha$, IL-1 $\beta$, IL-6, and IL-10 were measured to assess the inflammatory response. As shown in Figure 4, the levels of TNF- $\alpha$, IL-1 $\beta$, IL-6, and IL-10 were significantly increased in the LPS group compared with those in the control group (all $\mathrm{P}<0.05$ ). In the presence of LPS, cells transfected with VIM-siRNA exhibited increased release of these cytokines (all $\mathrm{P}<0.05$ ), while cells transfected with VIM-pCMV3 had decreased release of these cytokines $($ all $\mathrm{P}<0.05)$ in comparison to the control cells. There were no significant differences between the three groups in terms of TNF- $\alpha$, IL-1 $\beta$, IL-6, and IL-10 (all P>0.05).

\section{Effect on mitochondrial membrane proteins and proteins associated with apoptosis or autophagy}

As shown in Figure 5, the expression of mitochondrial membrane proteins (COXII, TOM20, and TIM 23) was significantly increased in the control cells in the presence of LPS (all $\mathrm{P}<0.05$ ). The LPS + VIM-pCMV3 vector had significantly decreased the levels of the mitochondrial membrane protein COXII compared with the level in the cells exposed to LPS (Figure 5, $\mathrm{P}<0.05$ ). Suppression of vimentin by siRNA plus LPS (LPS + VIM-siRNA) resulted in a further increase in these mitochondrial membrane proteins. There were no significant differences between the con, VIM-pCMV3, and VIM-siRNA groups (all P>0.05).

Regarding the expression of apoptosis-associated proteins, Bcl-2 expression was increased in the cells transfected with VIM-pCMV3 plus LPS compared to that in cells exposed only to LPS (Figure 5A,E, $\mathrm{P}<0.05$ ). However, it was decreased in the cells transfected with VIM-siRNA plus LPS compared to those exposed only to LPS (Figure 5A,E, $\mathrm{P}<0.05$ ). In contrast, caspase-3 was decreased in the cells transfected with VIM-pCMV3 plus LPS (Figure $5 A, F, \mathrm{P}<0.05$ ) but increased in the cells transfected with VIM-siRNA (Figure 5A,F, $\mathrm{P}<0.05$ ). There were no significant differences between the con, VIM-pCMV3, and VIM-siRNA groups (all P>0.05).

Similarly, the expression of the autophagy-associated proteins P62 and LC3II was also altered in the cells. P62 was significantly decreased in the cells transfected with VIM-pCMV3 plus LPS compared with that in the cells exposed to LPS (Figure $5 A, G, \mathrm{P}<0.05$ ). In contrast, the expression of LC3II was significantly increased in the cells transfected with VIM-pCMV3 plus LPS compared with 


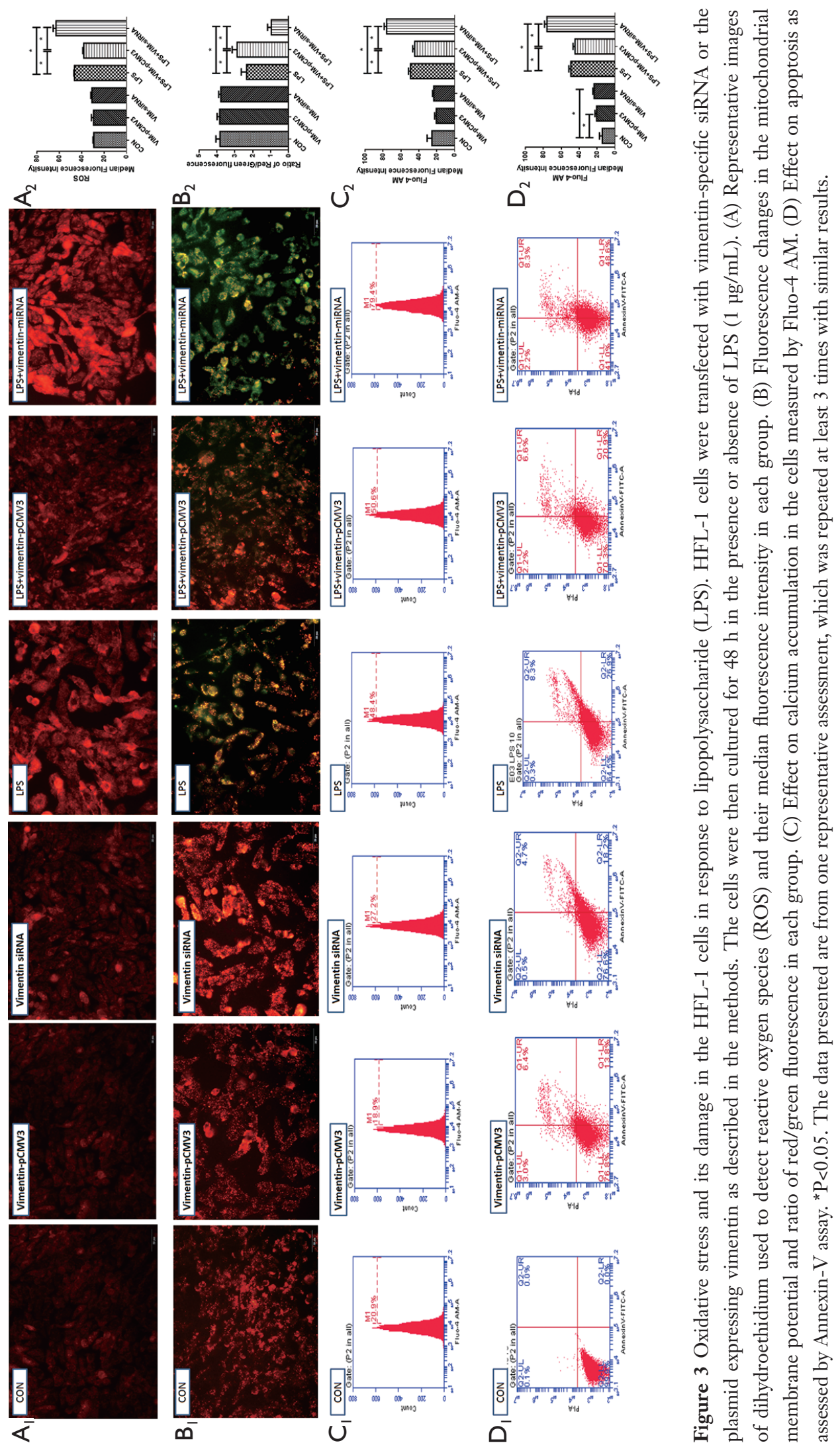




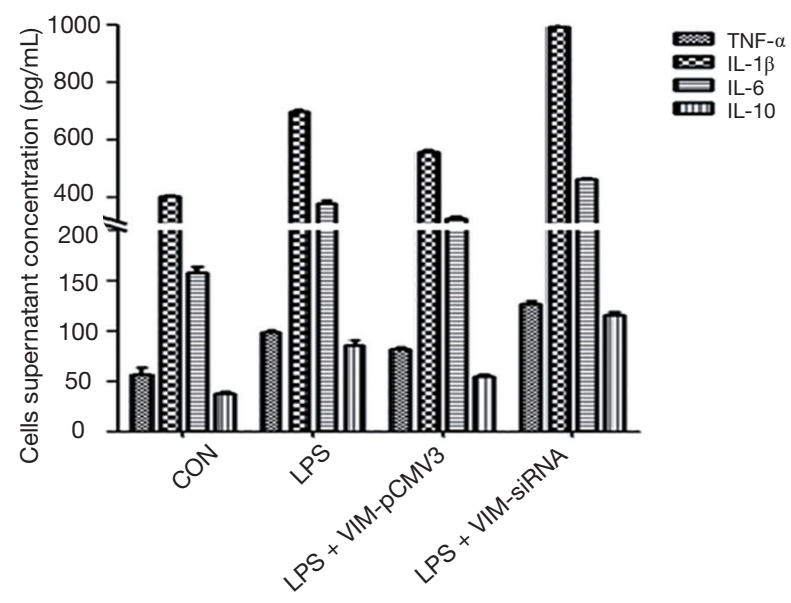

Figure 4 Release of cytokines by the HFL-1 cells in response to LPS. HFL-1 cells were transfected with vimentin-specific siRNA or a plasmid expressing vimentin as described in the method. The cells were then cultured in serum-free DMEM in the presence or absence of LPS $(1 \mu \mathrm{g} / \mathrm{mL})$ for $48 \mathrm{~h}$. Supernatants were harvested and used for quantification of the cytokines by ELISAs as described in the methods section. Vertical axes: cytokine amount expressed as $\mathrm{pg} / \mathrm{mL} / 10^{5}$ cells; horizontal axes: HFL-1 control cells, cells treated with LPS, cells treated with LPS after transfection of the plasmid expressing vimentin (VIM-pCMV3) or vimentin-specific siRNA (VIM-siRNA). The data presented are from one representative assessment, which was repeated 3 times with similar results. All data show statistically significant expression differences between each group $(\mathrm{P}<0.05)$.

that in the cells exposed only to LPS (Figure $5 A, G, \mathrm{P}<0.05$ ). There were no significant differences between the con, VIM-pCMV3, and VIM-siRNA groups (all $\mathrm{P}>0.05$ ).

\section{Discussion}

In this study, we reported that following the suppression of vimentin in human lung fibroblasts, cellular injury was aggravated by LPS intervention. Specifically, an increase in oxidative stress, a decrease in mitochondrial membrane potential, and an increase in calcium ion permeability resulted in an increase in the level of fibroblast apoptosis. Suppression of vimentin in lung fibroblasts also caused a decrease in autophagy, an increase in mitochondrial membrane protein, and a decrease in mitochondrial function, which might have contributed to the augmented cellular injury in response to LPS exposure (Figure 6).

When the lung is exposed to endogenous or exogenous factors, the defense mechanism is initiated. Alveolar macrophages are first activated, and a large number of inflammatory cells, especially neutrophils, are infiltrated, resulting in a large amount of ROS. Cells release inflammatory mediators, causing more inflammatory cells to aggregate, and inflammatory mediators can cause alveolar macrophages, alveolar epithelial cells, endothelial cells, and interstitial cells to produce more ROS, leading to "oxidative bursts" that cause lung tissue damage (2). Our previous study found that vimentin partially blocked the LPS-induced inflammatory response and the apoptosis of lymphocytes and monocytes, but the role of vimentin in interstitial lung cells and tissue damage was unknown. In this study, we observed that in the absence of vimentin, LPS increased the level of ROS in the lung fibroblasts, resulting in a decrease in cell membrane potential, a large influx of calcium ions, and an increased apoptosis rate. These findings suggested that ROS play important roles in mediating damage to tissue consisting of stromal cells, and vimentin may protect fibroblasts and reduce tissue damage in the early stage of ALI/ARDS.

Abnormal inflammatory responses play important roles in the development of ALI/ARDS, especially LPS-induced ALI/ARDS (3). The inflammatory response in ALI/ARDS is directly related to primary infections such as pneumonia and is also a pulmonary manifestation of systemic inflammation. Inflammatory responses and cytokine balance, as well as biological inhibitors and related molecules in the milieu of ALI, may be a key factor in the damage and repair of lung tissue (14). Inflammatory mediators can be secreted by activated cells recruited into the alveolar space under the inflammatory cascade or by dead cells (14). 

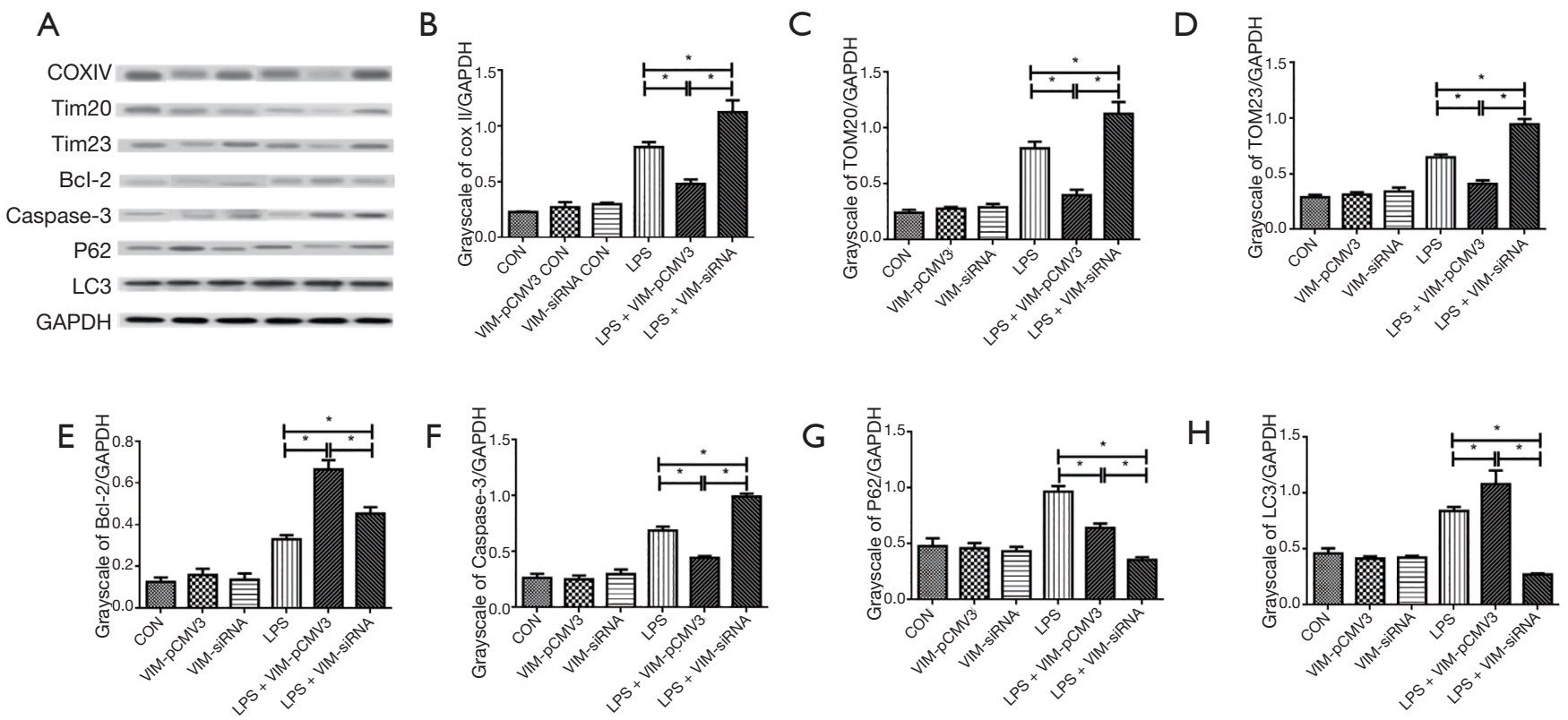

Figure 5 Expression of mitochondrial membrane proteins and apoptosis- or autophagy-associated proteins in the HFL-1 cells lacking vimentin or overexpressing vimentin. The HFL-1 cells were transfected with vimentin-specific siRNA or a plasmid expressing vimentin as described in the methods section. The cells were then cultured in serum-free DMEM in the presence or absence of LPS (1 $\mu \mathrm{g} / \mathrm{mL})$ for $24 \mathrm{~h}$. Total cell lysates were subjected to immunoblotting for COX II, TOM20, TIM 23, caspase-3, Bcl-2, and P62, with GAPDH as the loading control, as described in the methods section. (A) Representative immunoblotting. (B) Semiquantitative comparison of COX II. (C) Semiquantification of TOM20 expression. (D) Semiquantification of TIM23 expression. (E) Semiquantification of Bcl-2 expression. (F) Semiquantification of caspase-3 expression. (G) Semiquantification of P62 expression. (H) Semiquantification of LC3II expression. Vertical axes: grayscale of targeted proteins vs. GAPDH. Horizontal axes: HFL-1 control cells, cells treated with LPS, cells transfected with vimentin-specific siRNA (VIM-siRNA) or vimentin-expressing plasmid (VIM-pCMV3) plus LPS. Data are presented with significance denoted by ${ }^{*} \mathrm{P}<0.05$.

Interleukin (IL)- $1 \beta$ and TNF- $\alpha$ are the two most important proinflammatory cytokines in the early stage of ALI/ARDS, and both can promote pulmonary edema by activating endothelial cells and inducing ROS production, promoting inflammatory cell migration, and forming lung fibrosis. IL-6 and IL-10 constitute a pair of proinflammatory and anti-inflammatory cytokines, respectively. The imbalance of proinflammatory and anti-inflammatory factors may have particular importance in the regulation of lung tissue damage and repair. Our study found that IL- $1 \beta$, TNF- $\alpha$, and IL- 6 were increased while IL- 10 was decreased in the HFL-1 cells following vimentin suppression by siRNA and LPS exposure, suggesting that vimentin may be involved in the secretion of inflammatory mediators and cytokines in response to exposure to endotoxins.

Studies have shown that Nrf2 activation can regulate oxidative stress, xenobiotic metabolism and excretion, inflammation, apoptosis, autophagy, and cellular bioenergetics. Autophagy may have a protective role against cell damage (15). Mitochondria are the main organelles involved for the degradation of endogenous ROS in the cell through respiratory chain reactions. When mitochondrial autophagy is lost, intracellular ROS production increases, which causes intracellular damage (16). This study found that suppression of vimentin in lung fibroblasts led to a decrease in mitochondrial membrane protein and mitochondrial function in response to LPS, which might have been due to a decrease in autophagy function that resulted in a large accumulation of ROS and an increase in the apoptosis rate. Unexpectedly, we found that overexpression of vimentin by transfecting VIM-pCMV3 in lung fibroblasts also resulted in the decreased expression of the mitochondrial membrane protein. This outcome might have been due to mitophagy also influencing mitochondrial function. Nevertheless, the findings of the current study indicated that vimentin may play an important role in mediating the mechanism of lung 


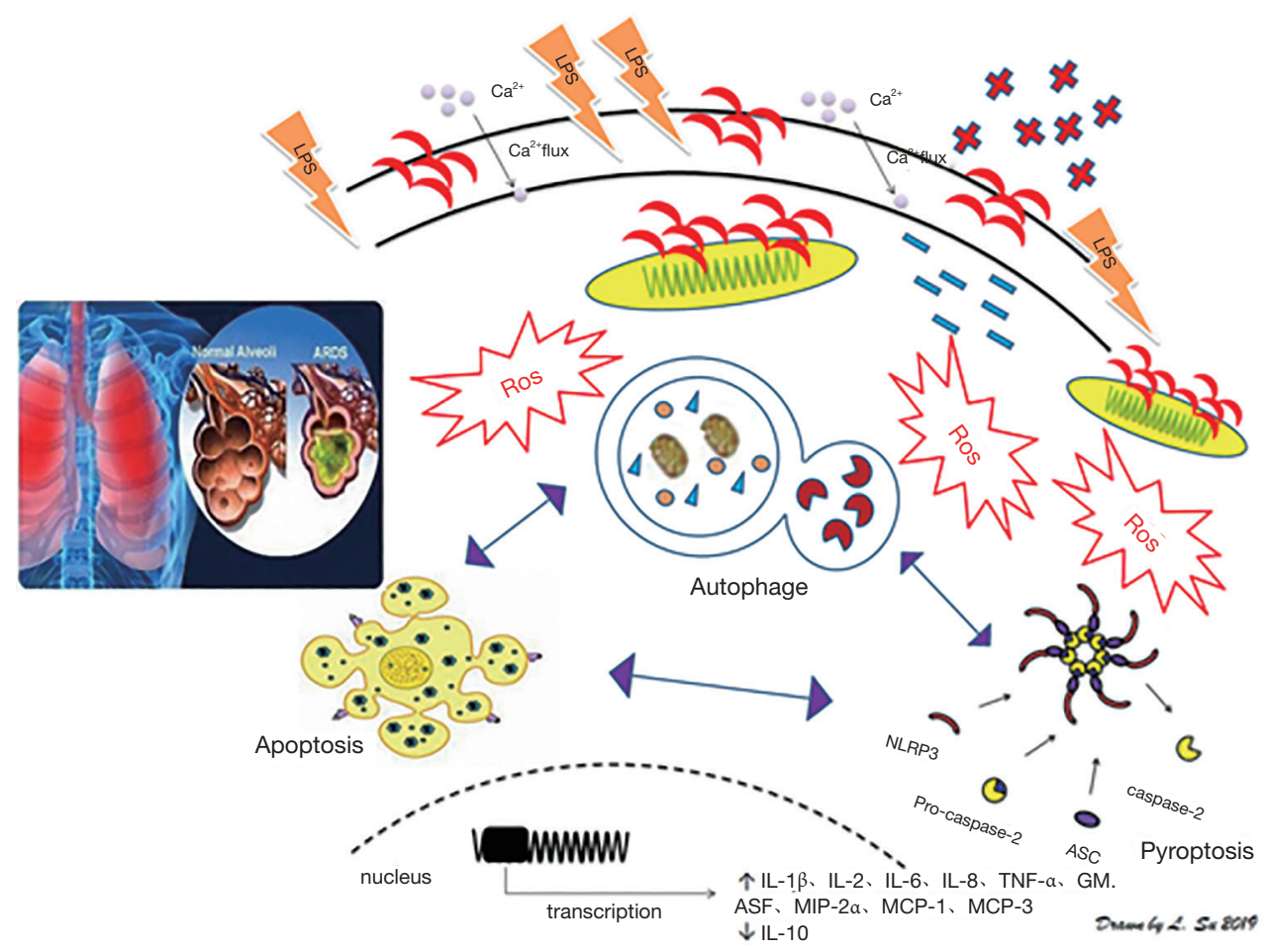

Figure 6 Vimentin regulation of the activation of autophagy in lung fibroblasts in response to LPS exposure based on an average of 3 separate experiments.

fibroblast apoptosis and autophagy in ALI/ARDS.

Oxidative stress and the inflammatory response are important components of the pathogenesis of ALI/ARDS $(17,18)$. The basis of ARDS pathogenesis is an excessive, uncontrolled, and amplified inflammatory response cascade. During the inflammatory response, parenchymal cells and interstitial cells of the lung can produce excessive ROS through respiratory bursts to cause oxidative stress and aggravate tissue damage. Vimentin can regulate the progression of the response to oxidative stress and inflammatory responses in HFL-1 cells the early stage of ALI/ARDS though the mitochondrial protection conferred by autophagy. However, the role of vimentin in the pulmonary fibrosis period or repair process may not be the same as it is in the early stage of ALI/ARDS.

Suppression of vimentin in lung fibroblasts resulted in increased mitochondrial oxidative stress and inflammatory responses but reduced autophagy function in the cells treated with LPS. In contrast, overexpression of vimentin partially protected these lung fibroblasts from oxidative stress-induced damage and autophagy dysfunction. The findings of the current study suggested that vimentin may interfere with the inflammatory cascade by activating the mitochondrial autophagy pathway in lung fibroblasts in the early stage of ALI.

\section{Acknowledgments}

Funding: The work in this paper was supported by the Beijing Nova Program (Grant No. Z201100006820126) from the Beijing Municipal Science \& the National Science Foundation for Young Scientists of China (Grant No. 81901935), Military Key Research Program (Grant No. BLB18J008).

\section{Footnote}

Reporting Checklist: The authors have completed the MDAR reporting checklist. Available at http://dx.doi.org/10.21037/ atm-20-5129

Data Sharing Statement: Available at http://dx.doi. org/10.21037/atm-20-5129

Conflicts of Interest: All authors have completed the ICMJE 
uniform disclosure form (available at http://dx.doi. org/10.21037/atm-20-5129). The authors have no conflicts of interest to declare.

Ethical Statement: The authors are accountable for all aspects of the work to ensure that questions related to the accuracy or integrity of any part of the work are appropriately investigated and resolved.

Open Access Statement: This is an Open Access article distributed in accordance with the Creative Commons Attribution-NonCommercial-NoDerivs 4.0 International License (CC BY-NC-ND 4.0), which permits the noncommercial replication and distribution of the article with the strict proviso that no changes or edits are made and the original work is properly cited (including links to both the formal publication through the relevant DOI and the license). See: https://creativecommons.org/licenses/by-nc-nd/4.0/.

\section{References}

1. Goss CH, Brower RG, Hudson LD, et al. Incidence of acute lung injury in the United States. Crit Care Med 2003;31:1607-11.

2. Kellner M, Noonepalle S, Lu Q, et al. ROS Signaling in the Pathogenesis of Acute Lung Injury (ALI) and Acute Respiratory Distress Syndrome (ARDS). Adv Exp Med Biol 2017;967:105-37.

3. Reiss LK, Schuppert A, Uhlig S. Inflammatory processes during acute respiratory distress syndrome: a complex system. Curr Opin Crit Care 2018;24:1-9.

4. Fan EKY, Fan J. Regulation of alveolar macrophage death in acute lung inflammation. Respir Res 2018;19:50.

5. Wang J, Liu YT, Xiao L, et al. Anti-inflammatory effects of apigenin in lipopolysaccharide-induced inflammatory in acute lung injury by suppressing COX-2 and NF-kB pathway. Inflammation 2014;37:2085-90.

6. Mor-Vaknin N, Punturieri A, Sitwala K, et al. Vimentin is secreted by activated macrophages. Nat Cell Biol 2003;5:59-63.

Cite this article as: Pan P, Su L, Wang X, Chai W, Liu D, Song L, Xie L. Vimentin regulation of autophagy activation in lung fibroblasts in response to lipopolysaccharide exposure in vitro. Ann Transl Med 2021;9(4):304. doi: 10.21037/atm-205129
7. Benes P, Maceckova V, Zdrahal Z, et al. Role of vimentin in regulation of monocyte/macrophage differentiation. Differentiation 2006;74:265-76.

8. dos Santos G, Rogel MR, Baker MA, et al. Vimentin regulates activation of the NLRP3 inflammasome. Nat Commun 2015;6:6574.

9. Su L, Pan P, Yan P, et al. Role of vimentin in modulating immune cell apoptosis and inflammatory responses in sepsis. Sci Rep 2019;9:5747.

10. Su LX, Pan P, Wang XT, et al. Vimentin modulates apoptosis and inflammatory cytokine release by a human monocytic cell line (THP-1) in response to lipopolysaccharides in vitro. Chin Med J (Engl) 2019;132:1336-43.

11. He Z, Gao Y, Deng Y, et al. Lipopolysaccharide induces lung fibroblast proliferation through Toll-like receptor 4 signaling and the phosphoinositide3-kinase-Akt pathway. PLoS One 2012;7:e35926.

12. He Z, Wang X, Deng Y, et al. Epigenetic regulation of Thy-1 gene expression by histone modification is involved in lipopolysaccharide-induced lung fibroblast proliferation. J Cell Mol Med 2013;17:160-7.

13. Meijerink J, Mandigers C, van de Locht L, et al. A novel method to compensate for different amplification efficiencies between patient DNA samples in quantitative real-time PCR. J Mol Diagn 2001;3:55-61.

14. Cross LJ, Matthay MA. Biomarkers in acute lung injury: insights into the pathogenesis of acute lung injury. Crit Care Clin 2011;27:355-77.

15. Rojo de la Vega M, Dodson M, Gross C, et al. Role of Nrf2 and Autophagy in Acute Lung Injury. Curr Pharmacol Rep 2016;2:91-101.

16. Gomes LC, Scorrano L. Mitochondrial morphology in mitophagy and macroautophagy. Biochim Biophys Acta 2013;1833:205-12.

17. Sarma JV, Ward PA. Oxidants and redox signaling in acute lung injury. Compr Physiol 2011;1:1365-81.

18. Tasaka S, Amaya F, Hashimoto S, et al. Roles of oxidants and redox signaling in the pathogenesis of acute respiratory distress syndrome. Antioxid Redox Signal 2008;10:739-53. 